\title{
Correction of metachromatic leukodystrophy in the mouse model by transplantation of genetically modified hematopoietic stem cells
}

\author{
Alessandra Biffi, ${ }^{1}$ Michele De Palma, ${ }^{1}$ Angelo Quattrini, ${ }^{2}$ Ubaldo Del Carro, ${ }^{2}$ Stefano Amadio, ${ }^{2}$ \\ Ilaria Visigalli, ${ }^{1}$ Maria Sessa, ${ }^{2}$ Stefania Fasano, ${ }^{3}$ Riccardo Brambilla, ${ }^{3}$ Sergio Marchesini, ${ }^{4}$ \\ Claudio Bordignon, ${ }^{1,5}$ and Luigi Naldini ${ }^{1,5}$
}

\begin{abstract}
${ }^{1}$ San Raffaele Telethon Institute for Gene Therapy; ${ }^{2}$ Neurology Department; ${ }^{3}$ Department of Molecular Biology and Functional Genomics, San Raffaele Scientific Institute, Milan, Italy. ${ }^{4}$ Department of Biomedical Science and Biotechnology, University of Brescia, Brescia, Italy. ${ }^{5}$ Vita Salute San Raffaele University, Milan Italy.
\end{abstract}

\begin{abstract}
Gene-based delivery can establish a sustained supply of therapeutic proteins within the nervous system. For diseases characterized by extensive CNS and peripheral nervous system (PNS) involvement, widespread distribution of the exogenous gene may be required, a challenge to in vivo gene transfer strategies. Here, using lentiviral vectors (LVs), we efficiently transduced hematopoietic stem cells (HSCs) ex vivo and evaluated the potential of their progeny to target therapeutic genes to the CNS and PNS of transplanted mice and correct a neurodegenerative disorder, metachromatic leukodystrophy (MLD). We proved extensive repopulation of CNS microglia and PNS endoneurial macrophages by transgene-expressing cells. Intriguingly, recruitment of these HSC-derived cells was faster and more robust in MLD mice. By transplanting HSCs transduced with the arylsulfatase A gene, we fully reconstituted enzyme activity in the hematopoietic system of MLD mice and prevented the development of motor conduction impairment, learning and coordination deficits, and neuropathological abnormalities typical of the disease. Remarkably, ex vivo gene therapy had a significantly higher therapeutic impact than WT HSC transplantation, indicating a critical role for enzyme overexpression in the HSC progeny. These results indicate that transplantation of LV-transduced autologous HSCs represents a potentially efficacious therapeutic strategy for MLD and possibly other neurodegenerative disorders.
\end{abstract}

\section{Introduction}

Metachromatic leukodystrophy (MLD) is a storage disorder that is due to inherited deficiency of the lysosomal enzyme arylsulfatase A (ARSA; EC 3.1.6.8). The disease is characterized by myelin degeneration in both CNS and peripheral nervous system (PNS), associated with the accumulation of undegraded galactosyl-3-sulfate ceramide (sulfatide) in glial cells and neurons. The prognosis is severe, with death resulting, in the majority of cases, a few years after the diagnosis $(1,2)$. No effective treatment is currently available. Protein therapy represents a powerful approach to replace a missing or nonfunctional enzyme, or to provide trophic and regulatory signals to affected tissues in a variety of metabolic, degenerative, or inflammatory conditions. However, protein delivery poses serious chal-

Nonstandard abbreviations used: arylsulfatase A (ARSA); bone marrow (BM); bone marrow transplantation (BMT); central conduction time (CCT); compound motor action potential (cMAP); cortical motor evoked potential (cMEP); glial fibrillary acidic protein (GFAP); hematopoietic stem cell (HSC); lentiviral vector (LV); $N$-lissamine rhodaminyl-(12-aminododecanoyl) cerebroside 3-sulfate (LRh-CS); metachromatic leukodystrophy (MLD); motor conduction velocity (MCV); motor evoked potentials (MEP); myelin basic protein (MBP); nervous system (NS); periodic acid-Schiff (PAS); peripheral conduction time (PCT); peripheral nervous system (PNS); phosphoglycerate kinase (PGK); PBS containing 1\% BSA and 0.1\% Triton X-100 (PBS-T); p-nitrocatecholsulfate assay (PNC); propidium iodide (PI); R-phycoerythrin (RPE); vesicular stomatitis virus (VSV); woodchuck hepatitis virus post-transcriptional regulatory element (Wpre)

Conflict of interest: The authors have declared that no conflict of interest exists.

Citation for this article: J. Clin. Invest. 113:1118-1129 (2004).

doi:10.1172/JCI200419205. lenges when sustained administration is required and when the CNS and PNS are the major disease targets, as in MLD. In fact, the blood-brain and the blood-nerve barriers may severely limit access of systemically administered therapeutic molecules to these tissues. Gene-based delivery may allow the establishment of a sustained source of therapeutic proteins within the nervous system (NS), overcoming the anatomical barriers that limit their diffusion from the circulation $(3,4)$. Others and we previously showed that direct injection of gene transfer vectors, or ex vivo-engineered cells, into the CNS achieved long-term protein expression and therapeutic benefit in several disease models, including MLD (5-9). However, the probable requirement for multiple injections and the invasiveness of the procedure may limit its application to humans. Moreover, the need to target the widespread PNS network poses an additional challenge. Thus, alternative strategies ensuring widespread delivery and expression of exogenous genes throughout the NS are required.

Following bone marrow transplantation (BMT), donor-derived cells have been detected within the CNS (10). The nature and fates of these CNS-migrated, bone marrow-derived (BM-derived) cells and their potential trans-differentiation into nonhematopoietic cells (i.e., neurons or astrocytes) has recently been the subject of extensive investigations (11-14). Several works supported the notion that donor BM-derived cells replaced a fraction of the CNS macrophage/microglia population in a probable process of physiological turnover and that this phenomenon was enhanced upon tissue damage $(15,16)$. By genetically marking the transplanted 
BM cells, marker expression was detected in the CNS macrophage/ microglia population, indicating that it is possible to deliver exogenous proteins to the CNS by genetically modified hematopoietic stem cells (HSCs) (17). The therapeutic potential of this approach is underscored by the clinical benefit of allogeneic BMT in some metabolic storage disorders $(18,19)$. Although the mechanism underlying the partial efficacy of BMT in controlling CNS disease is not fully understood, it is considered to reflect, at least in part, the secretion of functional enzyme by donor-derived cells that have migrated to the CNS and the subsequent enzyme uptake by the resident enzyme-deficient cells $(20,21)$. However, several aspects of the migration of BM-derived cells to the CNS remain to be better investigated, including their actual cellular source in the BM, their differentiation potential, kinetics, and extent of migration to different CNS regions in both physiological and pathological conditions. Furthermore, investigation is needed into whether a similar migration of BM-derived cells takes place in the PNS also and to what extent genetically modified HSCs can target the widespread PNS network. Most importantly, the potential therapeutic benefit of this strategy for disorders characterized by extensive CNS and PNS involvement such as MLD still requires demonstration. A major hurdle to overcome before exploiting HSC migration to reach sustained and effective levels of therapeutic proteins within the NS is to accomplish efficient gene transfer into the transplanted HSCs and stable, robust expression in their progeny. These are challenging tasks for the vectors currently in clinical studies (3).

Here we set out to address the aforementioned issues taking advantage of lentiviral vectors (LVs) to accomplish efficient HSC transduction in conditions that allow maintenance of stem cell properties and that may provide advantages for clinical application, as compared with currently used protocols (22-24). We transduced purified hematopoietic progenitors by a short ex vivo treatment in the absence of growth factors and monitored the fate of their gene-marked progeny into the NS upon transplantation and their potential to correct disease manifestations in As2 $2^{-/}$MLD mice, which carry germline inactivation of the ARSA gene (25). We confirmed and extended previous findings of progressive repopulation of CNS macrophage/microglia cells by transgene-expressing cells (17), proving the origin of CNS-migrated cells from long-term repopulating HSCs and their virtually exclusive differentiation into mature resting microglia. Moreover, we observed a faster and more pronounced migration of transgene-expressing macrophages into several PNS sites, providing direct evidence of PNS targeting by ex vivo modified cells. All of these findings were enhanced in MLD mice. Remarkably, when we transduced HSCs from MLD mice with ARSA-expressing LV and transplanted them into diseaseaffected littermates, we obtained full reconstitution of enzyme activity in the hematopoietic system and almost complete protection from the motor conduction impairments, motor learning and coordination deficits, sulfatide storage, and demyelination. These results indicate that transplantation of LV-transduced autologous HSCs may represent a long-sought therapeutic option for MLD patients, and possibly for other neurodegenerative disorders.

\section{Methods}

$L V$ production and titration. Vesicular stomatitis virus-pseudotyped (VSV-pseudotyped) LV stocks were produced by transient cotransfection of the transfer constructs pRRLsin.cPPT.hPGK.eGFP.Wpre (26) or PRRLsin.cPPT.hPGK.ARSA.Wpre, the late-generation packaging construct pCMVAR8.74, and the pMD2.G envelope construct in $293 \mathrm{~T}$ cells, followed by ultracentrifugation of conditioned medium, as described (27). Stocks were titered by Southern blot analysis, endpoint expression titer in HeLa cells, and p24 immunocapture assay, as described (28).

Mice studies. C57BL/6 and congenic C57BL/6 Ly45.1 mice were purchased from Charles River Laboratories Inc. (Calco, Italy) and maintained in germ-free conditions. As $2^{-/-}$MLD mice were bred in the H.S. Raffaele animal research facility by intercrossing the homozygous offspring of two carrier mice obtained by rederivation (embryo transfer) of As2 $2^{-/-}$males (25) with C57BL/ 6 females. Thus, the MLD mice used in this study have a mixed C57BL6/129 genetic background. WT C57BL6/129 hybrid mice were purchased from The Jackson Laboratory (Bar Harbor, Maine, USA) and used as the most appropriate controls for functional studies. All procedures were performed according to protocols approved by the Animal Care and Use Committee of the Fondazione San Raffaele del Monte Tabor (IACUC 163) and communicated to the Ministry of Health and local authorities according to Italian law.

Transduction of hematopoietic progenitors and BMT. Six-week-old male immunocompetent C57BL/6 Ly45.1 and As2 $2^{-/-}$mice were killed with $\mathrm{CO}_{2}$, and the $\mathrm{BM}$ was harvested by flushing the femurs and the tibias. Hematopoietic progenitors were purified using the Enrichment of Murine Hematopoietic Progenitors kit (Stem Cell Technologies Inc., Vancouver, British Columbia, Canada). For transduction, $1 \times 10^{6}$ cells $/ \mathrm{ml}$ were exposed to increasing doses of the phosphoglycerate kinase-GFP (PGK-GFP) LV (from $1 \times 10^{7}$ to $1 \times 10^{8} \mathrm{HeLa}$ transducing units/ml $[\mathrm{TU} / \mathrm{ml}])$ and PGK-ARSA LV (3 $\mu \mathrm{g}$ viral $\mathrm{p} 24 / \mathrm{ml})$ in Stem Span SFEM expansion medium (Stem Cell Technologies Inc.), in the absence of serum and cytokines for 12 hours. Vector- or mocktransduced cells $\left(10^{6}\right.$ cells/mouse) were injected via the tail vein into 6-week-old lethally irradiated (a total of 8 Gy divided into two administrations) C57BL/6 (Ly45.2) or As2 $2^{-/-}$female mice. We performed clonogenic assays by plating $1 \times 10^{4}$ and $5 \times 10^{4}$ hematopoietic progenitors in a methylcellulose-based medium (MethoCult M3434; Stem Cell Technologies Inc.). Ten days later, colonies were scored for GFP expression by fluorescence microscopy, plucked, and lysed for PCR analysis for the detection of LV sequences.

FACScan analysis. Transduced cells were grown for at least 4 days before FACS analysis to reach steady-state GFP expression and to rule out pseudo transduction. Before FACS analysis, cells were washed and resuspended in PBS containing $30 \mu \mathrm{M}$ propidium iodide (PI) (Becton Dickinson and Co., Franklin Lakes, New Jersey, USA) and $2 \%$ FBS. For immunostaining, $1 \times 10^{5}$ cells were blocked in $5 \%$ rat serum (Stem Cell Technologies Inc.), $2 \%$ FBS in PBS for 15 minutes at $4{ }^{\circ} \mathrm{C}$. After blocking, R-phycoerythrin-conjugated (RPE-conjugated) antibodies (IgG isotype control, anti-Sca-1 and anti-CD45.1, all from PharMingen, San Diego, California, USA) were added to a final concentration of $1-5 \mu \mathrm{g} / \mathrm{ml}$ and the cells incubated for 30 minutes at $4^{\circ} \mathrm{C}$, then washed, stained with PI, and analyzed by three-color flow cytometry. Only viable, PI-negative cells were used for the analysis.

To analyze engraftment, 12 and 24 weeks after transplant blood and marrow samples were subjected to immunostaining and FACS analysis following red blood cell lysis with ammonium chloride. Cells were stained as described above, with RPE-conjugated antibodies (IgG isotype control, anti-Sca-1, anti-CD45.1, anti-CD4, anti-CD8, anti-CD11b, and anti-B220, all from PharMingen) and analyzed.

PCR analysis. At sacrifice we performed clonogenic assays by plating $1 \times 10^{4}$ and $5 \times 10^{4} \mathrm{BM}$ cells in a methylcellulose-based medium (MethoCult M3434; Stem Cell Technologies Inc.). Ten days later, colonies were scored for GFP expression by fluorescence 
microscopy, plucked, and lysed for PCR analysis for the detection of LV sequences. Genomic DNA extracted from plucked hematopoietic colonies was subjected to PCR analysis for the HIV-1 leader region of the LV construct. GAPDH amplification was used to assess DNA integrity. Primers for the LV amplification were as follows: forward, 5'-TGAAAGCGAAAGGGAAACCA-3'; reverse, 5'CCGTGCGCGCTTCAG-3'. The PCR product length was $64 \mathrm{bp}$. Primers for GAPDH amplification were as follows: forward, $5^{\prime}$ CGCACTTTCTTGTGCAGTG-3'; reverse, $5^{\prime}$-GTTCAGCTCTCTGGGATGAC-3'. The PCR product length was $450 \mathrm{bp}$. The PCR reaction mixture consisted of $20 \mathrm{pmol}$ of each primer, $0.2 \mathrm{mM} \mathrm{dNTP}, 4$ $\mathrm{mM} \mathrm{MgCl}_{2}, 50 \mathrm{mM} \mathrm{KCl}, 10 \mathrm{mM}$ Tris- $\mathrm{HCl}$ ( $\mathrm{pH} 8.3$ ), and $1 \mathrm{U}$ AmpliTaq Gold DNA polymerase (Applied Biosystems, Foster City, California, USA). In the case of LV amplification, the first denaturation step was at $95^{\circ} \mathrm{C}$ for 10 minutes, followed by 30 cycles of 60 seconds at $94^{\circ} \mathrm{C}, 60$ seconds at $55^{\circ} \mathrm{C}, 60$ seconds at $72^{\circ} \mathrm{C}$, with the final step at $72^{\circ} \mathrm{C}$ for 5 minutes. In the case of GAPDH amplification, the first denaturation step was at $95^{\circ} \mathrm{C}$ for 10 minutes, followed by 30 cycles of 60 seconds at $94^{\circ} \mathrm{C}, 60$ seconds at $56^{\circ} \mathrm{C}, 60$ seconds at $72^{\circ} \mathrm{C}$, with the final step at $72^{\circ} \mathrm{C}$ for 5 minutes.

Southern blot analysis. The BM and the spleen of primary and secondary recipients were harvested for genomic DNA analysis. Twenty micrograms of genomic DNA and a curve of plasmid standards were digested with AflII, which cuts twice in the vector sequence, or with BamHI, which cuts once in the vector sequence. Digested DNA was separated on a $1 \%$ agarose gel, transferred to a nylon membrane (Hybond-N; Amersham Biosciences, Piscataway, New Jersey, USA) by capillary transfer, and probed with a radiolabeled woodchuck hepatitis virus post-transcriptional regulatory element (Wpre) probe.

Immunofluorescence and fluorescent microscope analysis. Animals were killed under deep anesthesia and by intracardiac perfusion with $0.9 \%$ $\mathrm{NaCl}$ followed by $4 \%$ paraformaldehyde (PAF) in PBS, $\mathrm{pH}$ 7.4. Organs were fixed for 10-12 hours in PAF, equilibrated for 48 hours in PBS containing $15 \%$ sucrose, and then embedded in OCT compound for quick freezing in liquid nitrogen. Six- to ten-micron cryostatic sections were laid on gelatin-coated slides, washed in PBS, and frozen at $-80^{\circ} \mathrm{C}$. For immunofluorescence staining, sections were blocked with $5 \%$ goat serum (Vector Laboratories Inc., Burlingame, California, USA) in PBS containing 1\% BSA and 0.1\% Triton X-100 (PBS-T). Primary antibodies were diluted in PBS-T with $2 \%$ goat serum, $1 \%$ BSA as follows: IgG2a K-isotype control clone A110-2, 1:200; F4/80 (MCAP497; Serotec Ltd., Oxford, United Kingdom), clone CI:A3-1, purified, 1:500; NeuN (MAB377; Chemicon International, Temecula, California, USA), 1:100; glial fibrillary acidic protein (GFAP) (MCA1909; Serotec Ltd.), clone DP46.109, 1:100; myelin basic protein (MBP) (MCA408; Serotec Ltd.), 1:100. For GFP staining we used an affinity-purified rabbit polyclonal antibody (A11122; Molecular Probes Inc., Eugene, Oregon, USA), 1:100. After incubation for 1 hour at room temperature, sections were washed in PBS-T and stained for 1 hour with secondary antibodies (goat $\alpha$-rabbit AlexaFluor488 and goat $\alpha$-rat AlexaFluor546; Molecular Probes Inc.) diluted 1:500 in PBS-T, 1\% BSA. For CD45.1 staining, we used an RPE-conjugated monoclonal anti-mouse CD45.1 antibody (A20; PharMingen), 1:50. Slides were mounted with 20\% Mowiol in PBS. Fluorescent and confocal microscopy were performed using a fluorescent microscope Olympus Provis AX70 and a three-laser confocal microscope (Radiance 2100; Bio-Rad Laboratories Inc., Hercules, California, USA). Fluorescent signals from single optical sections were sequentially acquired and analyzed by Adobe Photoshop 5.5 (Adobe Systems Inc., San Jose, California, USA).
Quantification of regional CNS and PNS engraftment. Counts of $\mathrm{GFP}^{+}, \mathrm{F} 4 / 80^{+}$cells in the CNS and in the PNS were performed on $10-\mu \mathrm{m}$ cryostatic sections following the immunofluorescence staining already described. Twenty to thirty brain (cerebrum and cerebellum) sections and 10-15 PNS (acoustic ganglion, dorsal root ganglions, and sciatic nerve) sections per mouse were analyzed from each of three mice per time point. As criteria for cell counts, only $\mathrm{GFP}^{+}, \mathrm{F} 4 / 80^{+}$cellular bodies, and not cellular processes, were counted. Counts were performed twice by two different investigators. An unpaired Student's $t$ test was performed for statistical evaluation of the data.

ARSA assays. To determine ARSA activity in transduced cells and in PBMC of ARSA-transplanted mice, cell pellets were lysed in 0.5 $\mathrm{M}$ sodium acetate, $\mathrm{pH} 5$, at $4^{\circ} \mathrm{C}$ for 2 hours. The ARSA activity was detected using the $\mathrm{N}$-lissamine rhodaminyl-(12-aminododecanoyl) cerebroside 3-sulfate (LRh-CS) as substrate (29), adapting the method for a pyrene containing cerebroside sulfate (30). The incubation mixtures, in final volumes of $0.25 \mathrm{ml}$, contained $25 \mathrm{mM}$ sodium acetate buffer ( $\mathrm{pH} 5.0$ ), $5.5 \mathrm{mM}$ sodium taurodeoxycholate, $5 \mathrm{mM} \mathrm{MnCl}_{2}, 50 \mu \mathrm{g}$ of sample proteins dialyzed against water, and $125 \mathrm{pmol}$ of LRh-CS. The samples and a blank were incubated at $37^{\circ} \mathrm{C}$ for 16 hours, stopped after addition of $0.5 \mathrm{ml}$ of $0.1 \mathrm{M} \mathrm{Na}_{2} \mathrm{SO}_{4}$, and adsorbed on a reverse-phase column (Sep-Pak C18 cartridge; Waters Corp., Milford, Massachusetts, USA); after exhaustive washing with water, the fluorescent lipids were recovered by elution with $2 \mathrm{ml}$ of methanol and $2 \mathrm{ml}$ of chloroform/methanol, 6:4. The combined eluates were evaporated under a nitrogen stream and resolved into bands by TLC on aluminum-coated silica gel plates using chloroform/ethylacetate/ n-propanol/0.25\% KCl/methanol, 25:25:25:9:16 (by volume). The spots corresponding to unreacted sulfatide, galactosyl cerebroside, ceramide, and free acid were scraped off the plate, extracted with chloroform/methanol, and their fluorescence measured on a Jasco FP-770 spectrofluorometer using an excitation wavelength of $565 \mathrm{~nm}$ and an emission of $575 \mathrm{~nm}$. ARSA activity was also evaluated by $p$-nitrocatecholsulfate (PNC) assay performed on $25 \mu \mathrm{g}$ of sample proteins incubated with $100 \mu \mathrm{l}$ of $10 \mathrm{mM}$ PNC for 90 minutes at $37^{\circ} \mathrm{C}$. The reaction was stopped with $1 \mathrm{ml}$ of $1 \mathrm{M} \mathrm{NaOH}$, and the fluorescence was measured in a spectrophotometer $(515 \mathrm{~nm})$. ARSA activity, expressed in nanomoles per milligram per hour, was normalized for protein content.

Neurophysiological evaluation. Mice were anesthetized with trichoroethanol, $0.02 \mathrm{ml} / \mathrm{g}$ of body weight, and placed under a heating lamp to avoid hypothermia. Using tape, we secured the mice on a smooth table to prevent movement artifacts due to the electrical stimulation, the lower limbs gently stretched to facilitate the measurement of distances between proximal and distal points of stimulation. The sciatic nerve motor conduction velocity (MCV) was obtained by stimulating the nerve with steel monopolar needle electrodes. A pair of stimulating electrodes was inserted subcutaneously near the nerve at the ankle; a second pair of electrodes was placed at the greater ischiatic notch to obtain two distinct sites of stimulation, distal and proximal, respectively, along the nerve. The muscular response to the electrical nerve stimulation, called the compound motor action potential (cMAP), was recorded with a pair of recording needle electrodes; the active electrode was inserted in muscles in the middle of the paw, while the reference was placed in the skin between the first and second digit. Motor evoked potentials (MEPs) were recorded with the same montage described for MCV from the paw muscle with transcra- 

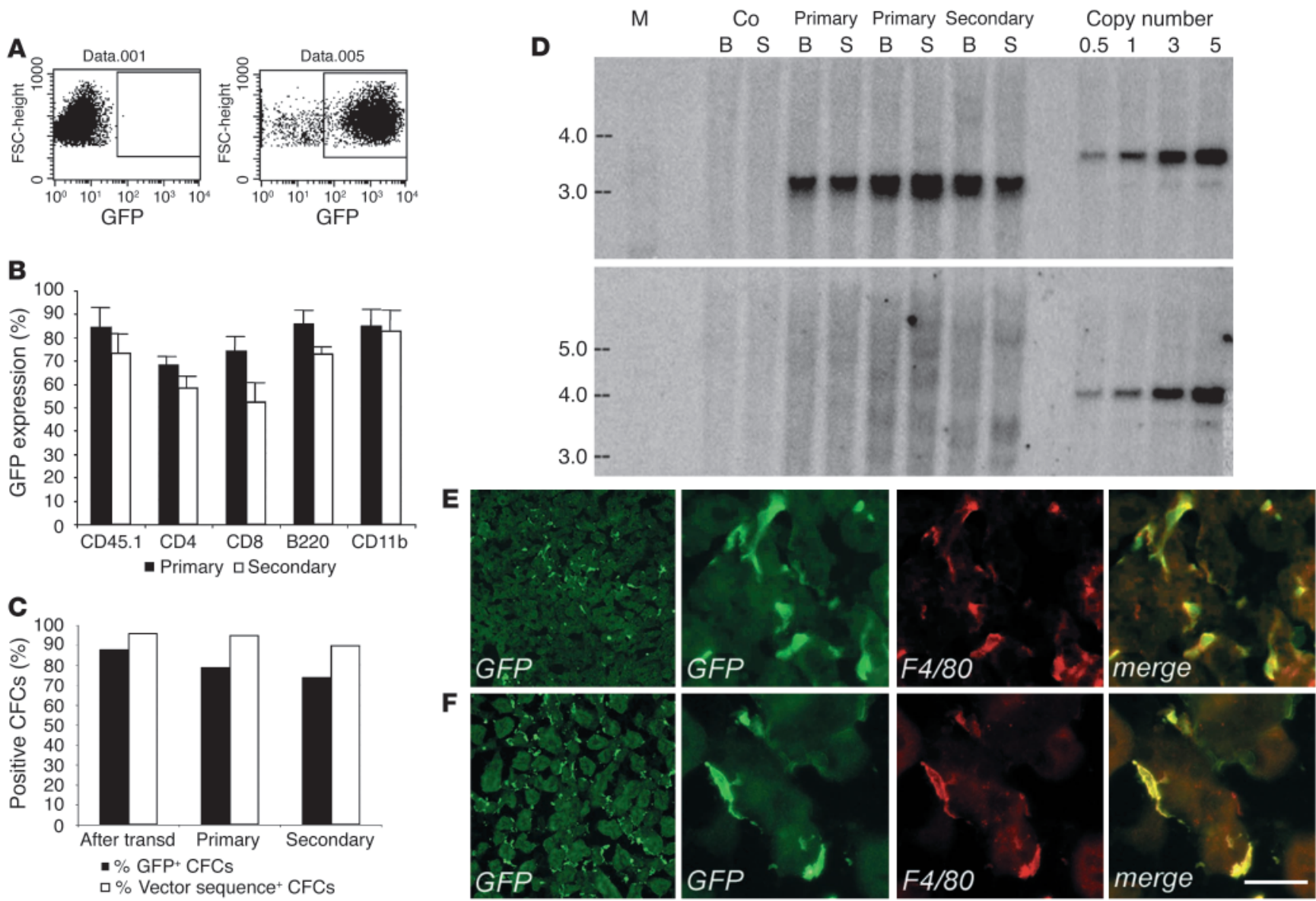

Figure 1

Chimerism and hematologic reconstitution by transgene-expressing cells of transplanted mice. (A) FACS analysis of GFP expression in control (left panel) and transduced (right panel) purified progenitors. (B) GFP expression in PBMCs of primary and secondary transplanted mice, 3 months after BMT. Up to $90 \%$ of circulating donor CD45.1-, CD11b-, B220-, CD4-, and CD8-positive cells expressed GFP. The means and standard deviations of primary $(n=16)$ and secondary $(n=4)$ mice are shown. (C) GFP expression by fluorescence microscopy $(\%$ GFP + and transduction level by PCR (\% vector sequence+), in CFCs from progenitors after transduction and from BM of primary and secondary recipients (50-100 CFCs scored for fluorescence and 20 CFCs analyzed by PCR per each mouse, $n=9$ ). The great majority of CFCs contained LV sequences and expressed GFP along serial transplants. (D) Southern blot analysis of bone marrow (B) and spleen (S) DNA from transplanted mice. A standard plasmid curve, from 0.5 to 5 vector copies per genome, DNA markers (M) in kb, and control untransplanted mice (Co) are shown. Aflll digestion (upper panel) showed 5 or more vector copies integrated per cell in all recipients. BamHI digestion (lower panel) showed a diffuse hybridization pattern in primary mice indicating oligo- to polyclonal engraftment and the absence of dominant clones. (E) and (F) GFP+ cells in liver (E) and kidney (F) sections of transplanted mice, 3 months after BMT, stained as indicated. GFP+ cells expressed F4/80 (3 panels on the right). Scale bar: $20 \mu \mathrm{m}$.

nial electrical stimulation of the motor cortex. The excitatory volleys descending along the corticospinal pathways evoke a motor potential in the paw muscles through a trans-synaptic depolarization of alpha-motor neurons (cortical MEP, cMEP). The peripheral conduction time (PCT) was obtained with a method based on the $\mathrm{F}$ wave latency determination. In fact, the antidromic volley following nerve stimulation excites the alfa-motor neuron, giving rise to spikes traveling orthodromically up to the muscle, where they can be recorded as a late potential called the F wave. The PCT was thus calculated with the formula $\left(\mathrm{F}_{\text {wave }} \mathrm{lat}+\mathrm{CMAP}_{\mathrm{lat}}-1\right) / 2$. The central conduction time (CCT) was measured as the difference between cMEP and PCT latencies. An unpaired Student's $t$ test was performed for statistical evaluation of the data.

Rotarod. A motor learning task was performed with an accelerating rotarod apparatus (Ugo Basile, Comerio, Italy). This rotarod equipment is based on a rotating cylinder, $3.2 \mathrm{~cm}$ in diameter, covered with textured rubber. Each section is $6.0 \mathrm{~cm}$ wide, allowing five mice to be tested simultaneously, one per section. Mice walk forward on the rotating cylinder, at speeds increasing from 4 to $40 \mathrm{rpm}$ over a 5 -minute test session. Latencies in falling off the cylinder were measured over 3 days, three trials per session. Statistical analyses were made by one-way ANOVA using Scheffe's test after significant main effect of the treatment was determined. Histopathology. Mice were killed by $\mathrm{CO}_{2}$ inhalation. The tissues were removed, fixed in $2 \%$ glutaraldehyde, $1 \%$ osmium tetroxide, and embedded in Epon/araldite. Semithin (1- $\mu$ m-thick) sections were stained with Toluidine Blue. Six to seven brain (cerebrum and cerebellum) and sciatic nerve sections per mouse were analyzed from each of two to four mice per condition. Metachromatic deposits larger than $20 \mu \mathrm{m}$ were counted at $\times 20$ magnification, demyelinated fibers were counted at $\times 100$ magnification. Counts were performed twice by two different investigators. Microscope 

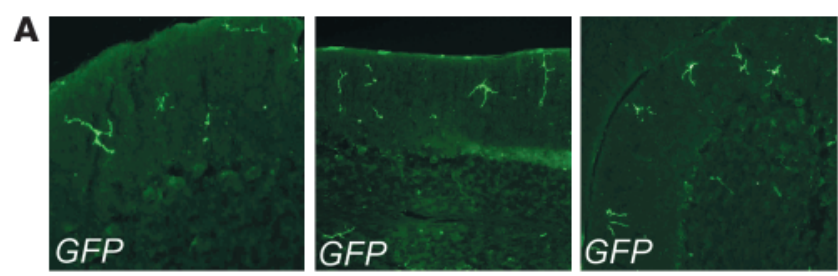

B
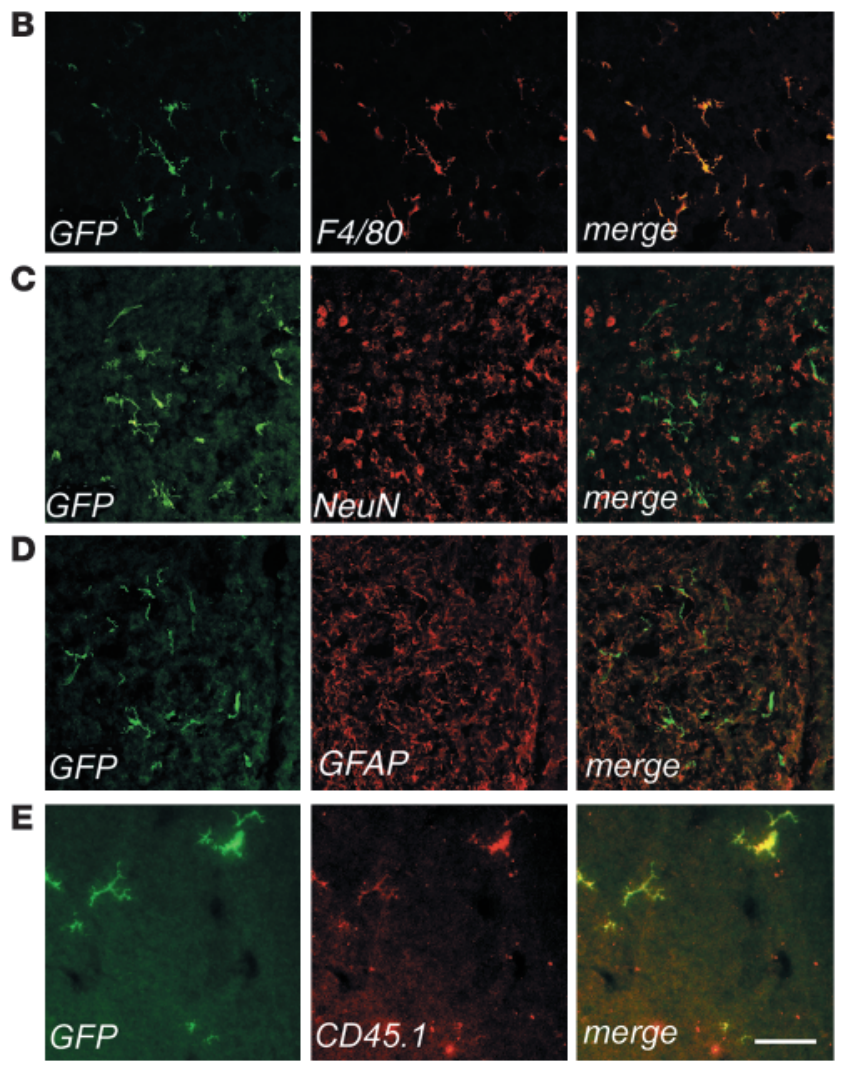

images were taken with a digital camera and processed by Adobe Photoshop 5.5 software (Adobe Systems Inc.).

\section{Results}

Long-term, multilineage, and polyclonal engraftment of LV-transduced HSCs. We enriched hematopoietic progenitors and stem cells from total BM of C57BL/6 Ly45.1 mice by lineage markers negative selection and transduced them using advanced-generation LV expressing the GFP marker gene under the control of the PGK promoter. To ensure full maintenance of stem cell properties, we incubated the

\section{Figure 2}

Identification of bone marrow-derived vector-expressing cells in the CNS of transplanted mice. Immunofluorescence analysis of cryostatic sections from the brains of transplanted mice. Fluorescent signals from single optical sections were sequentially acquired and are shown individually and after merging (merge). Immunostaining for GFP (green), F4/80, NeuN, GFAP, or CD45.1 (red) are indicated. (A) Representative sections from the cerebellums of transplanted mice, analyzed at 3 months (left panel, scale bar: $200 \mu \mathrm{m}$ ) and 6 months (middle and right panels, scale bar: 300 $\mu \mathrm{m}$ ) after BMT. Three months after BMT, few ramified cells were identified in the upper cortical layers near the meninges. By 6 months after BMT, several GFP+ ${ }^{+}$cells were present in the cortex, and small clusters of ramified GFP+ cells were detected throughout the parenchyma. (B-E) Representative brain sections from transplanted mice 9 months after BMT, immunostained as indicated. (B) GFP+ cells showed a ramified, microglial morphology and F4/80 immunoreactivity. Scale bar: $100 \mu \mathrm{m}$. (C and D) Overlay of GFP staining with the neuronal-specific marker NeuN (C) and the astrocytic marker GFAP (D) demonstrated separate localization of the two signals, with GFP+ ramified cells found between neurons and astrocytes. Scale bar: $300 \mu \mathrm{m}$. (E) CD45.1 immunostaining identified GFP+ cells as donor-derived. Scale bar: $70 \mu \mathrm{m}$.

cells for a short time with the vector in the absence of serum and cytokine stimulation (31). After transduction with optimized vector amounts $\left(0.5 \times 10^{8}\right.$ to $\left.1 \times 10^{8} \mathrm{TU} / \mathrm{ml}\right)$, up to $95 \%$ of the cells expressed the transgene, as assessed by GFP expression in liquid cultures (Figure 1A). To monitor chimerism by allele mismatch, we injected the transduced cells into lethally irradiated, congenic C57BL/6 Ly45.2 mice. FACS analysis of blood samples 3-9 months after the transplant demonstrated stable and high engraftment of Ly $45.1^{+}$donor cells (85-98\%, average $92 \%$, of total blood leukocytes; $n=16$ ), and sustained GFP expression levels (62-95\%, average $75 \%$, of total blood leukocytes; $n=16$ ) in the different hematopoietic cells lineages of recipient mice (Figure 1B). To verify self-renewal and long-term repopulating capacity of the transduced HSCs, we used the $\mathrm{BM}$ of primary recipients to perform secondary transplants. We found multilineage engraftment of transgene-expressing cells and GFP expression in $69 \%(53-85 \%, n=5)$ of the total blood leukocytes (Figure 1B) in the secondary-transplant mice 3 months after the transplant. PCR analysis and fluorescence microscopy of BMderived CFCs of primary and secondary recipients showed that the vast majority of the CFCs were positive for the vector sequences and expressed GFP (Figure 1C). Taken together, these results indicated that we efficiently transduced primitive long-term repopulating HSCs and that transgene expression was stable for up to 12 months in their differentiated progeny along serial transplants.

We then investigated the clonal composition and vector integration levels in the hematopoietic grafts of transplanted mice. South-

Table 1

Frequency of GFP+, F4/80+ macrophage/microglia in CNS and PNS of WT and As2--- mice

\begin{tabular}{|c|c|c|c|c|c|c|}
\hline & \multicolumn{3}{|c|}{ C57BL/6 mice } & \multicolumn{3}{|c|}{ As2--1- mice } \\
\hline & \multicolumn{2}{|c|}{ CNS } & \multirow[t]{2}{*}{ PNS } & \multicolumn{2}{|c|}{ CNS } & \multirow[t]{2}{*}{ PNS } \\
\hline & Ce & $\mathrm{Cb}$ & & $\mathrm{Ce}$ & $\mathrm{Cb}$ & \\
\hline 3 months, $n=3$ & $7.2 \pm 5.8$ & $11.5 \pm 6.1^{\mathrm{A}}$ & - & - & - & - \\
\hline 6 months, $n=3$ & $12.3 \pm 2^{B}$ & $13.4 \pm 3.2$ & $57 \pm 4.2$ & $21.5 \pm 1.65^{c}$ & $27.8 \pm 14.7^{c}$ & $90 \pm 6.7^{c}$ \\
\hline 9 months, $n=3$ & $23.9 \pm 6^{D}$ & $26.1 \pm 8.2^{D}$ & $69.7 \pm 13^{\mathrm{D}}$ & - & - & - \\
\hline
\end{tabular}




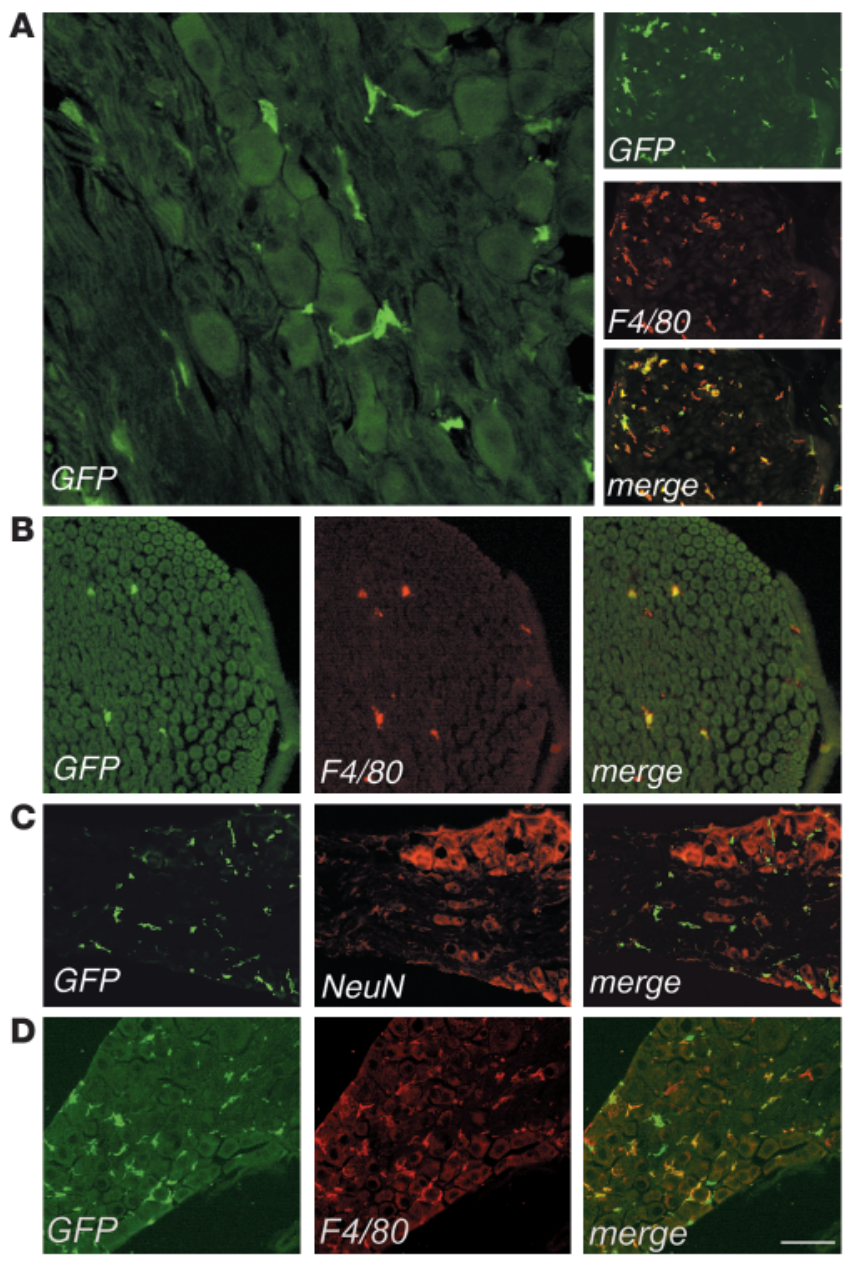

ern blot analysis on BM and spleen DNA, digested with AflII to measure the total vector integration, indicated that an average of five or more vector copies were integrated per cell in both primary and secondary recipients (Figure 1D). When the DNA was digested with BamHI to detect the individual vector integrations, we observed a diffuse hybridization pattern in most primary-transplant mice, indicating oligoclonal to polyclonal repopulation by HSCs carrying multiple vector integrations (Figure 1D). In secondary-

\section{Figure 3}

Identification of bone marrow-derived vector-expressing cells in the PNS of transplanted mice. Representative cryostatic sections of the dorsal root ganglion (A), sciatic nerve (B), and acoustic ganglion (C) of a transplanted mouse, 6 months after BMT, immunostained for GFP, F4/80, and NeuN, as indicated. (A) Left panel, GFP+ ${ }^{+}$cells were found in the dorsal root ganglion, surrounding sensory neurons and showing a macrophage morphology. Scale bar: $60 \mu \mathrm{m}$. Right panel, all of the $\mathrm{GFP}^{+}$cells coexpressed the macrophage marker F4/80. Scale bar: 200 $\mu \mathrm{m}$. (B) GFP+ cells were detected in the endoneurial space of the sciatic nerve and expressed F4/80. Scale bar: $100 \mu \mathrm{m}$. (C) GFP+ cells were distributed between sensory neurons in the acoustic ganglion and did not express the neuronal marker NeuN. Scale bar: $200 \mu \mathrm{m}$. (D) Vector-expressing cells in the PNS of a representative secondary transplant recipient. Cryostatic section from the dorsal root ganglion 4 months after BMT, immunostained for GFP and F4/80. Scale bar: $150 \mu \mathrm{m}$.

transplant mice, individual bands, which were barely detectable in a fraction of the primary-transplant mice, became more evident on a background of diffuse hybridization signal, indicating the presence of dominant clones and thus engraftment by a fraction of the primary repopulating HSCs (Figure 1D). These data indicated that, in our experimental conditions, HSCs were effectively preserved, as shown by the polyclonal repopulation of transplanted mice, and efficiently transduced, as shown by the high frequency of gene marking and by the multiple vector integrations into their progeny.

To better characterize our transplanted mice, we evaluated the migration of donor transduced HSC progeny into the visceral organs. We analyzed tissue sections of the liver, kidney, spleen, and lung 3 months after BMT, when the hematopoietic graft was fully established. We detected abundant $\mathrm{GFP}^{+}$cells in the liver with the morphology of Kupffer cells (Figure 1E), in the kidney with a branched morphology typical of macrophages (Figure 1F), in the red and white pulp of the spleen, and in the lung, at the level of alveoli (not shown). Colocalization studies demonstrated that the migrated $\mathrm{GFP}^{+}$cells nearly always coexpressed the macrophage markers F4/80 and/or CD11b, indicating that they turned over with the resident, tissue-specific monocyte/macrophage lineage population. These results showed a substantial contribution of transduced, donor-derived, hematopoietic lineage cells to the visceral organs of the recipient mice and stable vector expression in the extravascular progeny of the transplanted HSCs.

CNS migration of donor transduced HSC progeny and microglia differentiation. To evaluate the timing and the extent of migration of trans-

\section{Figure 4}

Enhanced migration and activated morphology of vector-expressing cells in the CNS of $A s 2^{--}$MLD mice. (A) Cryostatic sections of the corpus callosum of a representative transplanted MLD mouse 6 months after BMT, showing widespread GFP+ cells with a swollen ameboid morphology and coexpressing F4/80. Scale bar: $80 \mu \mathrm{m}$. (B) Cryostatic sections from the hippocampus of the same mouse were first immunostained as in $\mathbf{A}$, examined under the fluorescent microscope (three panels on the left) and then stained with PAS (panel on the far right). The GFP+, $\mathrm{F} 4 / 80^{+}$microglia cells were PAS-reactive, indicating their content of lipid storage granules. Scale bar: $40 \mu \mathrm{m}$.

\section{A}

A
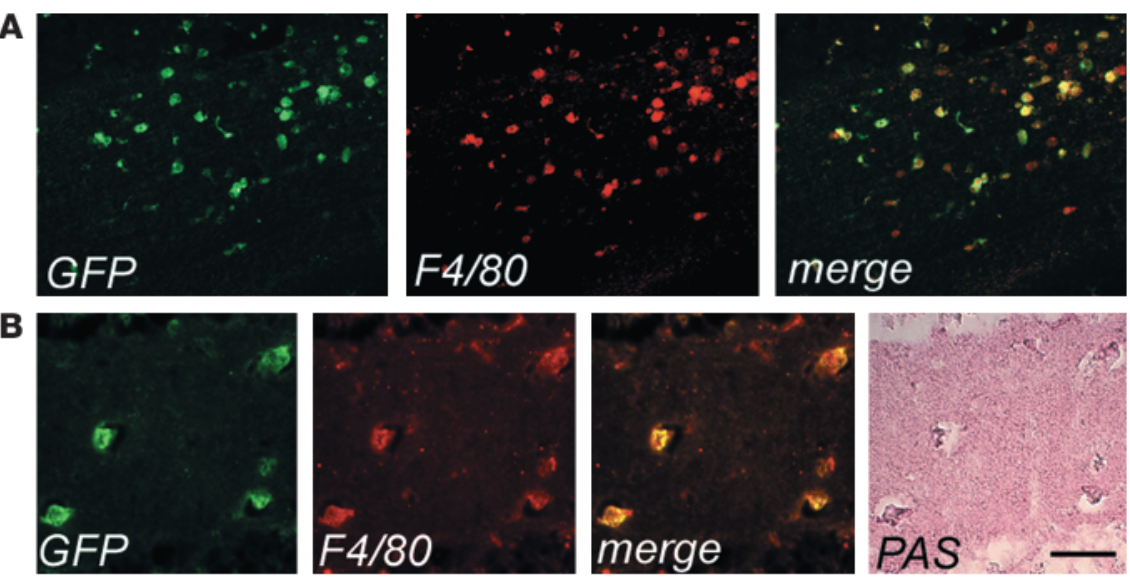


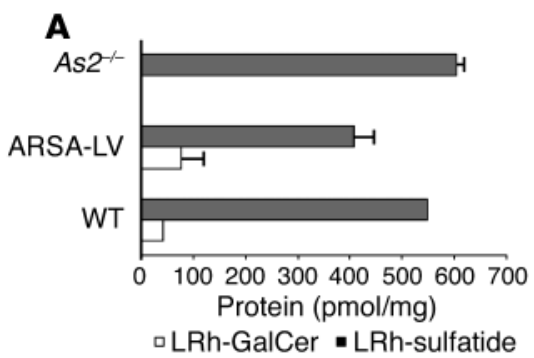

B

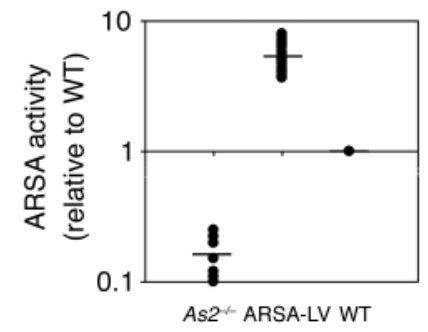

c

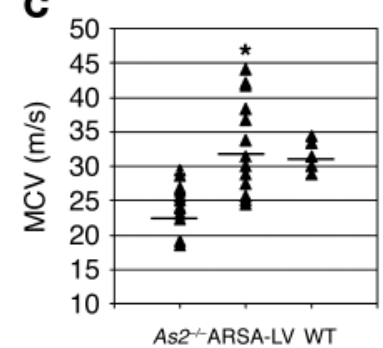

F

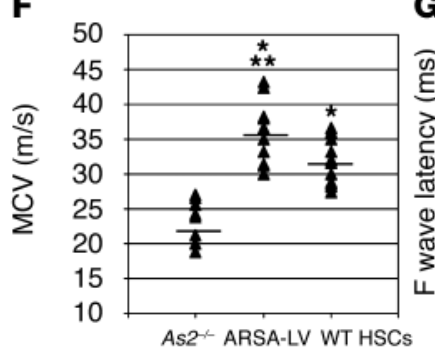

D

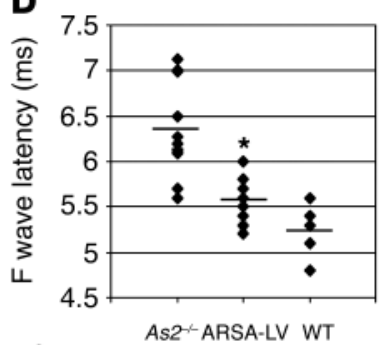

G

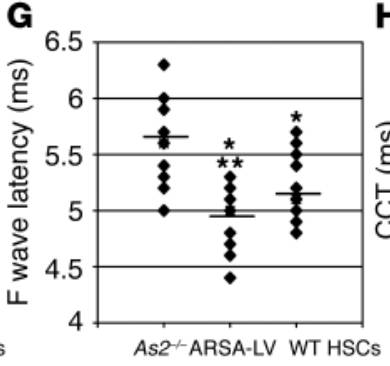

E

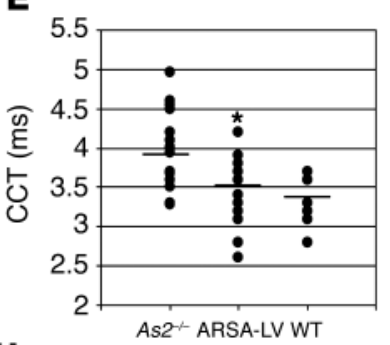

H

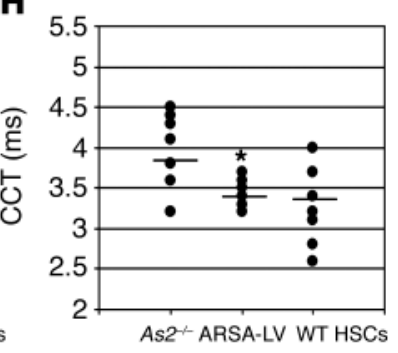

Figure 5

ARSA activity reconstitution and neurophysiological analysis of transplanted MLD mice. (A) Sulfatide assay on PBMCs from mice transplanted with ARSA-transduced (ARSA-LV) or GFP-transduced (As2--) HSCs, 7 months after BMT, and from WT mice. Sulfatide metabolism in WT and reconstituted $A s 2^{--}$mice is shown by LRh-sulfatide reduction and appearance of galactosylceramide (LRh-GalCer) (average and range of picomoles normalized for total protein content). (B) PNC assay on PBMCs as in A. ARSA activity is expressed relative to the value obtained from WT mice. Full ARSA activity reconstitution and overexpression above the WT level was observed in ARSA-transplanted mice. (C-E) Neurophysiological assessment of central and peripheral motor conduction in 8-month-old MLD mice transplanted with ARSA- or GFP-transduced HSCs, and WT mice with the same genetic background ( $n=15$ mice per group). Significantly lower CCTs (C) and F wave latency values (D), and significantly higher sciatic MCV values (E) were recorded in ARSA- transplanted mice compared with GFP controls. Comparison with WT shows nearly complete prevention of motor conduction impairments $(P>0.05$ for all parameters). ( $\mathbf{F}-\mathbf{H})$ Neurophysiological assessment in the same groups of ARSA- and mock-transplanted mice as in C-E, at 12 months of age, and in age-matched, WT HSC-transplanted mice ( $n=10$ mice per group). The analysis shows maintenance of the therapeutic effect in ARSA-transplanted mice and significantly faster motor conduction as compared with WT HSC-transplanted ones $\left({ }^{\star} P<0.01\right.$ between ARSA-LV and As2 ${ }^{--;} ;{ }^{* \star *} P<0.05$ between ARSA-LV and WT HSC-transplanted mice). Data are expressed as single recordings and means.

duced, donor-derived cells to the host CNS, we analyzed sections from different brain regions of transplanted mice at 3,6, and 9 months after BMT by fluorescence and confocal microscopy for expression of GFP and cell-specific markers (Figure 2). At 3 months after the transplant, several GFP-expressing cells were detected in the cerebrum and in the cerebellum. GFP ${ }^{+}$cells with a ramified morphology, characteristic of microglia, were mainly detected in the outer cortical layers near the meninges (Figure 2A), while less differentiated cells were detected throughout the brain in association with blood vessels. All of these CNS-migrated cells strongly expressed GFP, as determined by direct fluorescence (not shown) and by immunofluorescence staining using anti-GFP antibody. Immunostaining and confocal analysis revealed that almost all $\mathrm{GFP}^{+}$cells expressed the monocyte/macrophage-specific F4/80 marker (shown as example in Figure 2B). Interestingly, a higher frequency of ramified cells was found in the cerebellar parenchyma at the earlier time of analysis after BMT. By 6 and 9 months after BMT, we observed a significant increase $(P<0.01$ for both time points) in the frequency of ramified $\mathrm{GFP}^{+}$cells, both in the cerebrum and cerebellum (Figure 2, A and B, and Table 1). At the final time of analysis, up to $30 \%$ of the resident $\mathrm{F} 4 / 80^{+}$microglia were $\mathrm{GFP}^{+}$ in the cerebrum and in the cerebellum. At this long-term analysis, the $\mathrm{GFP}^{+}, \mathrm{F} 4 / 80^{+}$cells were often found in clusters in the different regions of the CNS analyzed, including the neocortex, hippocampus, fimbria, cerebellar cortex, vermis, and spinal cord. The vast majority of these cells showed a ramified morphology, identifying them as mature, resting microglia (Figure 2B). Moreover, the $\mathrm{GFP}^{+}$cells in the CNS were negative for NeuN and astrocyte-specific (GFAP) markers in all examined sections (Figure 2C, D). To further confirm the donor and hematopoietic origin of CNS-migrated cells, we performed double immunofluorescence for GFP and the CD45.1 antigen, which was solely expressed by donor-derived cells. We found that nearly all the $\mathrm{GFP}^{+}$cells expressed CD45.1 (Figure 2E), and that CD45.1 expression was higher in less differentiated, perivascular cells and lower in welldifferentiated, parenchymal microglia cells, as previously reported (30). Moreover, these CD45.1 $1^{+}, \mathrm{GFP}^{+}$cells did not express the host CD45.2 marker (not shown), thus excluding the concept that GFP expression was due to uptake of GFP-containing debris from circulating or extravasated cells by host macrophages/microglia. In addition, the lack of CD45.2 expression by $\mathrm{GFP}^{+}$cells ruled out any significant role of cell fusion in the observed microglia reconstitution by HSC-derived cells. These results confirmed the origin of CNS-migrated $\mathrm{GFP}^{+}$cells from donor HSCs and their virtually exclusive differentiation into mature, resting microglia. We then analyzed the CNS of secondary transplant recipients, and we found $\mathrm{GFP}^{+}$cells in the cerebrum, cerebellum, and spinal cord with a frequency similar to that observed in primary recipients (data not shown). As in primary hosts, transduced cells showed a ramified morphology and were positive for F4/80 expression. This further confirmed that CNS-migrated cells originated from self-renewing, long-term repopulating HSCs.

PNS migration of donor transduced HSC progeny. We then analyzed sections from the acoustic and dorsal root ganglions and from the sciatic nerve of transplanted mice by fluorescent and confocal 


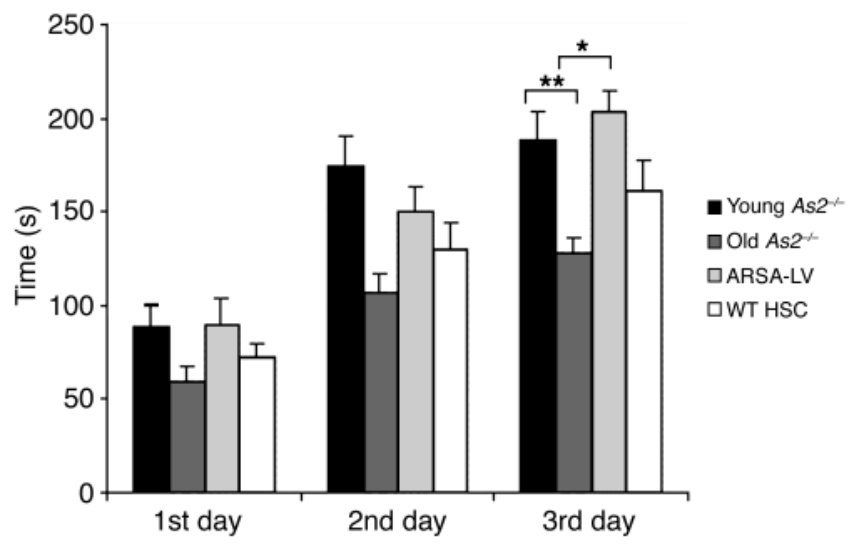

Figure 6

Motor learning and coordination in treated and untreated MLD mice. Twelve-month-old mice transplanted with ARSA-transduced or WT HSCs and two cohorts of untreated As2 ${ }^{--}$mice of 3 and 12 months of age were tested on an accelerating rotarod apparatus ( $n=12-30$ per group). Latencies to fall off the rotarod were recorded over 3 days, 3 consecutive trials per day. Means \pm SEM of each day are indicated. Post hoc comparisons were made by one-way ANOVA using Scheffe's test after significant main effect of the treatment was determined. Differences from 12-month-old untreated mice were considered significant at $P<0.05$ $\left({ }^{\star} P<0.01 ;{ }^{*} P<0.001\right)$. The decline in motor learning and coordination was completely prevented by ARSA-transduced HSC transplantation, whereas WT HSC transplantation was not significantly effective in preventing the development of the motor learning impairment.

microscopy for the expression of GFP and cell-specific markers (Figure 3). Starting 3 months after BMT, we found several $\mathrm{GFP}^{+}$cells in all the PNS sites examined (Figure 3A). Immunostaining and confocal analysis showed that almost all of the $\mathrm{GFP}^{+}$cells expressed the monocyte/macrophage-specific F4/80 marker (Figure 3, A and B). The $\mathrm{GFP}^{+}$cells were found between the somata of sensory neurons in the ganglia and within the endoneurium in the sciatic nerve, expressed F4/80, and were negative for NeuN (Figure 3C) and for the Schwann cell-specific MBP marker (data not shown), thus being identified as endoneurial macrophages. Six months after the transplant, $\mathrm{GFP}^{+}$cells accounted for $55-60 \%$ of the $\mathrm{F} 4 / 80^{+}$cells in sensory ganglions (acoustic and dorsal root ganglions) and sciatic nerve (Table 1). Nine months after BMT, we observed a further increase in the frequency of $\mathrm{GFP}^{+}$cells in the PNS, reaching $77 \%$ and $62 \%$ of the total $\mathrm{F} 4 / 80^{+}$cells in sensory ganglions and sciatic nerve, respectively, and thus approaching the levels observed in circulating monocytes. These data indicated an extensive migration of transduced HSC progeny to the PNS occurring with a faster kinetics than that observed in the CNS. As in primary hosts, we found several $\mathrm{GFP}^{+}$cells in the sciatic nerve and in the acoustic and dorsal root ganglions of secondary-transplant recipients, showing distribution and surface marker expression similar to those observed in primary-transplant mice (Figure 3D and other data not shown). Since the transplanted committed progenitors do not self-renew over the long term in the host and since donor chimerism of the BM stroma is not expected in mice transplanted with purified hematopoietic progenitors, the observed CNS and PNS migration of transgeneexpressing cells in secondary-transplant recipients proved their origin from ex vivo-transduced, primitive HSCs.

CNS and PNS migration in MLD mice. We then investigated the migration of donor transduced HSC progeny to the CNS and PNS of As2--
MLD mice. Others and we previously described the accumulation of nonmetabolized sulfatide in metachromatic granules in the white matter, and the glial and neuronal degeneration occurring in selected regions of the NS of these mice $(3,25)$. We marked purified hematopoietic progenitors from $A s 2^{-1-}$ mice with the PGK-GFP LV and injected them into lethally irradiated $A s 2^{-1-}$ recipients. As observed in the C57BL/ 6 transplants described above, we found GFP expression in $69-95 \%$ (average $82 \%, n=9$ ) of all blood lineages 3 months after transplant (data not shown). We detected an extensive migration of $\mathrm{GFP}^{+}, \mathrm{F} 4 / 80^{+}$cells in both the CNS and PNS of $A s 2^{-/-}$mice analyzed 6 months after BMT. The transduced, donor-derived cells were particularly abundant in white matter-rich regions that are preferentially affected by lipid storage, such as the corpus callosum and the hippocampal fimbria (Figure 4). Interestingly, virtually all $\mathrm{GFP}^{+}, \mathrm{F} 4 / 80^{+}$ cells in these disease target sites showed the swollen, ameboid morphology of activated microglia (Figure 4) $(32,33)$ and periodic acid-Schiff (PAS) reactivity due to sulfatide storage in the cytoplasm (Figure 4B). The contribution of donor HSC-derived, transduced cells to the local microglial population was significantly higher $(P<0.01)$ than that observed in normal C57BL/ 6 mice at the same time point after BMT (Table 1). Similarly, we found abundant $\mathrm{GFP}^{+}$cells in the PNS and a higher frequency of these cells with respect to that observed in WT mice (Table 1). These data indicated enhanced recruitment from the BM and/or faster turnover of resident macrophage/ microglia population in the NS of MLD mice and preferential targeting of lipid storage sites by transgene-expressing activated microglia. Taken together, they suggest that transplantation of LV-transduced autologous HSCs may represent a promising gene therapy strategy for MLD, with the potential to correct an early and most likely critical target of the disease, such as the macrophage/microglia, and deliver the therapeutic enzyme throughout the CNS and PNS.
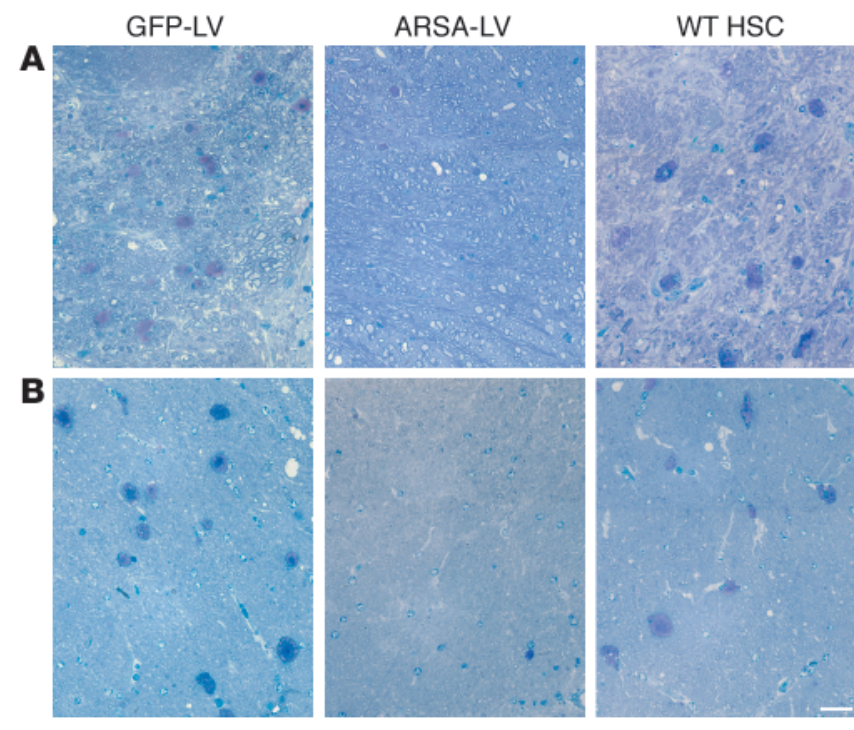

\section{Figure 7}

Long-term protection from lipid storage in transplanted MLD mice. (A and $\mathbf{B}$ ) Toluidine Blue-stained semithin sections of the cerebellum (A) and the hippocampal fimbria (B) of representative 12-month-old MLD mice transplanted with GFP-LV- and ARSA-LV-transduced As2 ${ }^{-/-}$ HSCs, or with WT HSCs. Abundant large metachromatic deposits are present in mock-treated and WT HSC-transplanted mice, whereas they are almost absent in mice transplanted with gene-corrected cells. Scale bar: $50 \mu \mathrm{m}$. For quantitative data and sample size, see Table 2 . 


\begin{tabular}{|c|c|c|c|c|}
\hline $\begin{array}{l}\text { Transplanted } \\
\text { HSC }\end{array}$ & $\begin{array}{l}\text { Sciatic nerve } \\
\text { Deposits }\end{array}$ & $\begin{array}{c}\text { Cerebellum } \\
\text { Demyelination }\end{array}$ & $\begin{array}{l}\text { Fimbria } \\
\text { Deposits }\end{array}$ & Deposits \\
\hline$A s 2^{-/-}$ & +++ & + & +++ & ++++ \\
\hline GFP-LV & +++ & + & ++++ & ++++ \\
\hline WT 1 & + & + & +++ & ++ \\
\hline WT 2 & ++ & + & ++++ & +++ \\
\hline ARSA-LV 1 & $+/-$ & - & + & + \\
\hline ARSA-LV 2 & $+/-$ & - & + & ++ \\
\hline ARSA-LV 3 & - & - & $+/-$ & $+/-$ \\
\hline ARSA-LV 4 & - & - & $+/-$ & $+/-$ \\
\hline
\end{tabular}

Neuropathological grading: -, 0; +/-, 1-4; +, 5-10; ++, 6-12; +++, 13-20; ++++, >20 metachromatic deposits $\geq 20 \mu \mathrm{m}$ per microscope field or section at $\times 20$ magnification in cerebellum and fimbria, and of metachromatic deposits in Schwann cells and demyelinated fibers per nerve section at $\times 100$ magnification in the sciatic nerve; $n=6$ sections per mouse examined for each site.

ARSA activity reconstitution in transplanted MLD mice. To assess the therapeutic potential of gene-corrected HSC transplantation in preventing disease progression in the CNS and PNS, we transduced ARSA-deficient HSCs from MLD mice with LV expressing the human ARSA cDNA (3) or the control GFP marker and transplanted them into disease-affected littermates. Transduction efficiency was evaluated by LV-specific PCR on CFCs obtained from the transduced HSCs, as previously described. Up to $90 \%$ of the CFCs were positive for the LV sequence (not shown). ARSA activity reconstitution in the transduced HSC progeny was evaluated after in vitro liquid culture by substrate metabolism. The cerebroside 3-sulfate assay showed complete reconstitution of sulfatide metabolism in ARSA-LV-transduced As2 $2^{-/-}$HSCs (not shown). PNC assay demonstrated that ARSA activity in the transduced cells reached values 5- to 10-fold higher than those measured in cells from WT controls (not shown). Seven months after BMT, transplanted MLD mice were analyzed for ARSA activity in PBMCs. By sulfatide assay, we observed reconstitution of ARSA-dependent sulfatide metabolism, shown by the breakdown of input sulfatide and the production of its direct metabolite galactosylceramide (Figure 5A). ARSA specific activity was quantified as fourto eightfold higher than that observed in WT mice (Figure 5B). These data demonstrated full, sustained reconstitution of the specific enzyme activity in the hematopoietic system of transplanted mice to levels significantly higher than those observed in WT mice.

Protection from motor conduction abnormalities in transplanted $M L D$ mice. At 4, 7, and 11 months after BMT, when transplanted mice were 5, 8, and 12 months old, respectively, we performed electroneurographic recordings and MEP. We found a statistically significant difference $(P<0.01)$ between mice transplanted with ARSA-transduced HSCs and GFP-transduced HSCs in MCV (Figure 5, C and F), F wave latency (Figure 5, D and G) (the latter two parameters being evaluated on the sciatic nerve), spinal and cortical MEP latencies (not shown), and CCT (Figure 5E and H). Remarkably, MLD mice transplanted with gene-corrected HSCs reached the normal range of each tested parameter, as determined by performing the same measurements in 8-month-old WT mice with a comparable genetic background (C57BL/6-SV129 hybrids) (Figure 5, C-E). These data demonstrated long-lasting and almost complete protection from motor conduction impairments in MLD mice transplanted with gene-corrected HSC. Similar evaluations performed 11 months after the transplant on mice transplanted with As2 $2^{--}$ARSA-transduced HSCs or WT HSCs demonstrated a statistically significant advantage of the gene therapy approach, as shown by better preserved peripheral conduction $(P<0.05$ for MCV and F wave latency) (Figure 5, F and G). Moreover, WT HSC-transplanted mice did not reach a significant increase in CCT because of high variability in the response to treatment (Figure $5 \mathrm{H}$ ).

Protection from motor learning and coordination impairment in transplanted MLD mice. Mice transplanted at 12 months of age with either ARSA-transduced HSCs or WT HSCs, and two groups of untreated MLD mice, 3 and 12 months of age, respectively, were subjected to the rotarod test to assess motor learning and coordination (34) (Figure 6). Two-way ANOVA revealed a significant effect of ARSA-transduced HSC transplantation $(P<0.05)$. Post hoc comparison revealed a significant deterioration of motor learning and coordination in 12-month-old, as compared with 3-month-old, untreated MLD mice $(P<0.001)$, consistent with previous reports documenting the late onset of the disease phenotype in the mouse model (5, 25). Remarkably, the motor learning and coordination impairment was fully prevented by ARSA-transduced HSC transplantation (ARSA-transduced HSC vs. untreated 12-month-old mice, $P<0.01$; ARSA-transduced HSC vs. untreated 3-month-old mice, $P=0.99$ ). As in the case of neurophysiological evaluation, transplantation of WT HSCs, though showing a trend toward improving mouse performance, did not reach a statistically significant effect (WT HSC vs. untreated 12 -month-old mice, $P=0.52$ ).

Protection from neuropathology in transplanted MLD mice. We then performed histopathological analysis on the CNS and PNS of transplanted mice at 12 months of age. We observed a marked reduction of sulfatide-containing metachromatic granules in the white matter of the brain and cerebellum of ARSA-transplanted mice (Figure 7 and Table 2), as compared to age-matched untreated or control vector-treated mice. On the contrary, mice trans-

\section{Figure 8}

Long-term protection from lipid storage and demyelination in transplanted MLD mice. Toluidine Blue-stained sections of the sciatic nerve of representative 12-month-old MLD mice transplanted with GFP-LV - and ARSA-LV-transduced $A s 2^{-/-}$HSCs, or with WT HSCs. Several demyelinated fibers (arrows) and metachromatic granules in Schwann cells are present in mock-treated and WT HSC-transplanted mice, whereas they are almost absent in mice transplanted with gene-corrected cells. Scale bar: $40 \mu \mathrm{m}$. For quantitative data and sample size, see Table 2.
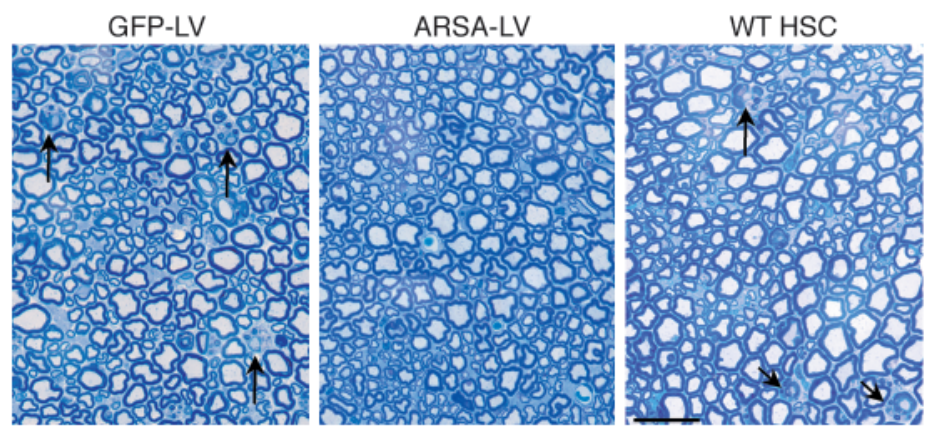
planted with WT HSCs showed abundant metachromatic deposits in the disease target areas (Figure 7 and Table 2). Moreover, in ARSA-transplanted mice, we observed a reduction in the activated, ameboid microglia, with the vast majority of microglia in lipid storage sites showing a resting and well-differentiated morphology (not shown). Consistently with the conduction impairments, we found demyelinated fibers and abundant metachromatic deposits in the sciatic nerve of untreated and mock-treated MLD mice (Figure 8 and Table 2). Mice transplanted with ARSA-transduced cells showed not only a marked reduction in sulfatide deposits in the sciatic nerve, but also an almost complete protection from demyelination (Figure 8 and Table 2). Metachromatic deposits and demyelinated fibers were instead present in the sciatic nerve of mice transplanted with WT HSC (Figure 8 and Table 2). These data indicate that ARSA-LV-transduced HSC transplantation provides an effective protection from the disease-associated tissue damage. Moreover, they suggest a critical role of enzyme overexpression in the HSC progeny, given the limited efficacy of WT HSC transplant.

\section{Discussion}

In this work, we demonstrate the potential of genetically modified HSCs to target gene therapy to the NS and correct MLD in the As2 $2^{-/-}$ mouse model. We used LV and optimized conditions to achieve efficient gene transfer into HSCs, robust, long-term gene expression in their progeny, and polyclonal repopulation of transplanted mice, all of which are potentially critical advantages in prospective clinical applications. We showed extensive reconstitution of CNS microglia and PNS macrophages by the transgene-expressing progeny of longterm repopulating HSCs. By transplanting HSCs transduced with the ARSA gene, we fully reconstituted enzyme activity in the hematopoietic system of MLD mice and prevented the development of the motor conduction impairments, learning and coordination deficits, and neuropathological abnormalities typical of the disease.

We proved the origin of NS-migrated cells from long-term repopulating HSCs and their virtually exclusive differentiation into microglia in the CNS, and into macrophages in the PNS. In the brain, the frequency of transgene-expressing cells increased progressively with time, as was recently reported in mice transplanted with retroviral vector-transduced whole BM cells (17), suggesting continuous recruitment from circulating precursors and making it unlikely that radiation-induced tissue damage before BMT was solely responsible for this phenomenon. This hypothesis is further supported by our preliminary results obtained in mice transplanted after busulfan conditioning (35), in which we confirmed an extensive migration of donor HSC-derived cells into the recipient NS (A. Biffi et al., unpublished observations). Transgene-expressing cells were mainly perivascular and poorly differentiated at earlier times after BMT, while at later times an increasing fraction of them showed the ramified morphology and the widespread CNS distribution of mature resting microglia $(33,36)$. These results are consistent with physiological turnover of resting CNS microglia by recruitment of circulating precursors. They undermine earlier beliefs that the perivascular subset of microglia is the only one to be periodically replaced by BM-derived elements (37), rather suggesting that perivascular cells may represent an intermediate stage in the process of monocyte differentiation to ramified mature microglia. Interestingly, we observed a faster rate of appearance of transgene-expressing cells in the cerebellum, possibly reflecting regional diversity of the blood-brain barrier between supratentorial and subtentorial structures not only in pathological (38) but also in physiological conditions. It remains to be determined whether the
CNS-recruited cells underwent local proliferation or were often found in clusters because of regional variation in the recruitment.

Remarkably, we observed an even more pronounced migration of transgene-expressing macrophages into several different PNS sites, indicating an unexpected turnover rate of endoneurial macrophages via $\mathrm{BM}$ recruitment. The frequency of transgene-expressing monocyte lineage cells found in these PNS sites approached that observed in the BM and peripheral blood when analyzed 6 months after BMT, indicating a faster turnover of endoneurial macrophages than that of CNS microglia, and possibly reflecting the more permeable bloodnerve barrier. The function of endoneurial macrophages in physiological conditions is unclear, although they are known to patrol tissues and provide an early response to damage $(39,40)$. Their unexpectedly high frequency and turnover, as revealed by our genemarking studies, may also indicate a role in the normal disposal of nerve components (41). Whatever their role is in the PNS, significant recruitment of BM-derived macrophages provides an avenue for vehicle gene therapy to this widespread and hardly accessible tissue.

The potential value of the CNS and PNS gene delivery strategy proposed here was further highlighted by our observation of faster and enhanced recruitment of BM-derived transgeneexpressing cells in the NS of MLD mice. The rapid response of hematogenous macrophages to acute local injury and inflammation is well known and was previously reported for the CNS after focal cerebral ischemia $(17,42)$ and for the PNS after sciatic nerve crush injury (43). The mechanisms underlying our findings in the chronic, slowly progressive neuropathology of MLD mice are less clear, and the implications of our observations may be relevant for understanding the disease pathogenesis and developing new therapeutic strategies. In fact, the abundant microglia recruited from the BM to brain areas particularly affected by lipid storage showed the typical morphology of activated microglia and was a preferential target of lipid accumulation. The presence of astrogliosis and microglial activation with few signs of demyelination has been previously reported in the NS of MLD mice (25). These data are of particular interest given the recent reassessment of the pathogenetic role of microglia in storage CNS disorders $(33,44)$. Activated microglia have been proposed to trigger neuronal and glial cell death by cytokine release induced by their inability to process the stored lipid. Importantly, the gene delivery approach described here corrected the metabolic deficiency, and probably the pathogenetic role, of a primary disease target, as shown by the significant reduction in the frequency of activated microglia cells in the CNS of MLD mice transplanted with ARSA gene-transduced HSCs. It remains to be ascertained whether the gene-corrected microglia cells have a survival advantage over their ARSA-deficient counterparts in the CNS; if they do, this could possibly contribute to a faster and more effective reconstitution upon BMT. In addition, the genecorrected microglia could become a local source of the therapeutic enzyme for other resident NS populations. Cross-correction of enzyme-deficient cells has been well demonstrated and constitutes the basis for enzyme replacement therapy and allogeneic BMT in several lysosomal storage disorders (21). Although crosscorrection of ARSA-deficient murine oligodendrocytes was previously demonstrated in vitro (45), whether and to what extent it also occurs in vivo in the experimental settings reported here will require formal demonstration (see below and also ref. 46).

By performing detailed neurophysiological studies of MLD mice, we found significant impairments of central and peripher- 
al motor conduction (MCV reduction, increased $\mathrm{F}$ wave, $\mathrm{MEP}$ and CCT latencies), which provide useful functional parameters to monitor disease progression and therapeutic efficacy, and were not evaluated or assessable in previous studies $(3,25,47,48)$. Behavioral tests demonstrated a clear decline in motor learning and coordination in aging MLD mice. Moreover, histopathological analysis demonstrated, in addition to the previously described sulfatide storage throughout the CNS and PNS $(5,25)$, demyelination in the sciatic nerve. These abnormalities correlate well with the functional and behavioral impairments and closely match the human pathology.

LV-mediated HSC transduction and transplantation restored ARSA activity in vivo to supranormal levels because of multiple vector integrations. Remarkably, ARSA overexpression in the HSC progeny led to almost complete prevention of the motor conduction and behavioral impairments. Histopathological analysis showed protection from microglia activation, sulfatide accumulation, and demyelination in the NS. These results suggest that ARSA overexpression not only corrected the metabolic defect in microglia cells, which have been directly implicated in disease pathogenesis, but may also have provided a source of active enzyme for other cell types in the NS.

Previous transplantation studies using retroviral vector-transduced BM cells failed to observe significant benefits in NS pathology and functional parameters in MLD mice $(47,48)$. It is likely that the successful outcome of our approach was dependent on the efficient HSC transduction and robust gene expression in their vascular and extravascular progeny reached by the optimized LVs and conditions used in this study. Direct comparison with MLD mice transplanted with WT HSCs showed that the HSC-based gene ther- apy approach was the only one achieving clear-cut efficacy, highlighting the crucial role of enzyme overexpression and indicating the probable occurrence of in vivo cross-correction. The unique benefit here achieved by genetically corrected versus WT cells provides a strong rationale for a future clinical translation of HSCbased gene therapy in MLD. Further support comes from the poor clinical outcome of allogeneic BMT in $\operatorname{MLD}$ patients $(18,19)$, although the complexity of the immunological donor-recipient interplay in allogeneic BMT is likely to account for significant differences between inbred mice and patients, and by the advantages of an autologous versus allogeneic HSC source. Further studies will address the safety of LV-mediated HSC gene therapy, and its possible application to other neurodegenerative disorders.

\section{Acknowledgments}

We are indebted to I. Bertani, L. Sergi-Sergi, G. Dina, A. Fanzani, and S. Grassi for technical help and to A. Consiglio and T. Luca for helping to establish the MLD colony. This research was supported by grants from Telethon, EU (QLK3-1999-00859), Associazione Italiana per la Ricerca sul Cancro (23-2001) to L. Naldini, and from the Italian Ministry of Scientific Research to L. Naldini and R. Brambilla.

Received for publication June 16, 2003, and accepted in revised form January 20, 2004.

Address correspondence to: Luigi Naldini, San Raffaele Telethon Institute for Gene Therapy, via Olgettina 58, 20132 Milan, Italy. Phone: 39-02-2643-4681; Fax: 39-02-2643-4668; E-mail: naldini.luigi@hsr.it.
1. Kolodney, E.H., and Fluharty, A.L. 1995. Metachromatic leukodystrophy and multiple sulfatase deficiency: sulfatide lipidosis. In The metabolic and molecular bases of inherited disease. 6 th edition. C.R. Scriver, A.L. Beaudet, W.S. Sly, and D. Valle, editors. McGraw-Hill, New York, USA. 2693-7391.

2. Neufeld, E.F. 1991. Lysosomal disease. Annu. Rev. Biochem. 60:257-280.

3. Kay, M.A., Glorioso, J.C., and Naldini, L. 2001. Viral vectors for gene therapy: the art of turning infectious agents into vehicles of therapeutics. Nat. Med. 7:33-40.

4. Glorioso, J.C., Mata, M., and Fink, D.J. 2003. Therapeutic gene transfer to the nervous system using viral vectors. J. Neurovirol. 9:165-172.

5. Consiglio, A., et al. 2001. In vivo gene therapy of metachromatic leukodystrophy by lentiviral vectors: correction of neuropathology and protection against learning impairments in affected mice. Nat. Med. 7:310-316.

6. Bosch, A., Perret, E., Desmaris, N., Trono, D., and Heard, J.M. 2000. Reversal of pathology in the entire brain of mucopolysaccharidosis type VII mice after lentivirus-mediated gene transfer. Hum. Gene Ther. 11:1139-1150.

7. Kordower, J.H., et al. 1999. Lentiviral gene transfer to the nonhuman primate brain. Exp. Neurol. 160:1-16.

8. Taylor, R.M., and Wolfe, J.H. 1997. Decreased lysosomal storage in the adult MPS VII mouse brain in the vicinity of grafts of retroviral vector-corrected fibroblasts secreting high levels of beta-glucuronidase. Nat. Med. 3:771-774.

9. Bachoud-Levi, A.C., et al. 2000. Neuroprotective gene therapy for Huntington's disease using a polymer encapsulated BHK cell line engineered to secrete human CNTF. Hum. Gene Ther. 11:1723-1729.

10. Hickey, W.F., Vass, K., and Lassmann, H. 1992. Bone marrow-derived elements in the central nervous system: an immunohistochemical and ultrastructural survey of rat chimeras. J. Neuropathol. Exp. Neurol. 51:246-256.

11. Brazelton, T.R., Rossi, F.M.V., Keshet, G.I., and Blau, H.M. 2000. From marrow to brain: expression of neuronal phenotypes in adult mice. Science. 290:1775-1779.

12. Mezey, E., Chandross, K.J., Harta, G., Maki, R.A., and McKercher, S.R. 2000. Turning blood into brain: cells bearing neuronal antigens generated in vivo from bone marrow. Science. 290:1779-1782.

13. Mezey, E., et al. 2003. Transplanted bone marrow generates new neurons in human brains. Proc. Natl. Acad. Sci. U. S. A. 100:1364-1369.

14. Weimann, J.M., Charlton, C.A., Brazelton, T., Hackman, R.C., and Blau, H.M. 2003. Contribution of transplanted bone marrow cells to Purkinje cells in human adult brains. Proc. Natl. Acad. Sci. U. S. A. 100:2088-2093.

15. Eglitis, M.A., and Mezey, E. 1997. Hematopoietic cells differentiate into both microglia and macroglia in the brains of adult mice. Proc. Natl. Acad. Sci. U. S. A. 94:4080-4085.

16. Kennedy, D.W., and Abkowitz, J.L. 1997. Kinetics of central nervous system microglial and macrophage engraftment. Analysis using a transgenic bone marrow transplantation model. Blood. 90:986-993.

17. Priller, J., et al. 2001. Targeting gene-modified hematopoietic cells to the central nervous system: use of green fluorescent protein uncovers microglial engraftment. Nat. Med. 7:1356-1361.

18. Krivit, W., Aubourg, P., Shapiro, E., and Peters, C. 1999. Bone marrow transplantation for globoid cell leukodystrophy, adrenoleukodystrophy, metachromatic leukodystrophy, and Hurler syndrome. Curr. Opin. Hematol. 6:377-382.

19. Peters, C., and Steward, C.G. 2003. Hematopoietic cell transplantation for inherited metabolic diseases: an overview of outcomes and practice guidelines. Bone Marrow Transplant. 31:229-239.

20. Krivit, W., Sung, J.H., Shapiro, E.G., and Lockman, L.A. 1995. Microglia: the effector cell for reconstitution of the central nervous system following bone marrow transplantation for lysosomal and peroxisomal storage diseases. Cell Transplant. 4:385-392.

21. Neufeld, E.F., and Muenzer, J. 2001. Lysosomal storage disorders. In The metabolic and molecular bases of inherited disease. C.R. Scriver, A.L. Beaudet, W.S. Sly, and D. Valle, editors. McGraw-Hill. New York, New York, USA. 3421-3452.

22. Ailles, L., et al. 2002. Molecular evidence of lentiviral vector-mediated gene transfer into human self-renewing, multi-potent, long-term NOD/SCID repopulating hematopoietic cells. Mol. Ther. 6:615-626.

23. Guenechea, G., Gan, O.I., Dorrell, C., and Dick, J.E. 2001. Distinct classes of human stem cells that differ in proliferative and self-renewal potential. Nat. Immunol. 2:75-82.

24. Miyoshi, H., Smith, K.A., Mosier, D.E., Verma, I.M., and Torbett, B.E. 1999. Transduction of human $\mathrm{CD} 4^{+}$cells that mediate long-term engraftment of NOD/SCID mice by HIV vectors. Science. 283:682-686.

25. Hess, B., et al. 1996. Phenotype of arylsulfatase A-deficient mice: relationships to human metachromatic leukodystrophy. Proc. Natl. Acad. Sci. U. S. A. 93:14821-14826.

26. Follenzi, A., Ailles, L.E., Bakovic, S., Geuna, M., and Naldini, L. 2000. Gene transfer by lentiviral vectors is limited by nuclear translocation and rescued by HIV-1 pol sequences. Nat. Genet. 25:217-222.

27. Follenzi, A., and Naldini, L. 2002. HIV-based vectors. Preparation and use. Methods Mol. Med. 69:259-274.

28. De Palma, M., and Naldini, L. 2002. Transduction of a gene expression cassette using advanced generation 
lentiviral vectors. Methods Enzymol. 346:514-529.

29. Marchesini, S., et al. 1990. Synthesis, spectral properties and enzymatic hydrolysis of fluorescent derivatives of cerebroside sulfate containing long-wavelength-emission probes. Chem. Phys. Lipids. 53:165-175.

30. Marchesini, S., Viani, P., Cestaro, B., and Gatt, S. 1989. Synthesis of pyrene derivatives of cerebroside sulfate and their use for determining arylsulfatase A activity. Biochim. Biophys. Acta. 1002:20-27.

31. De Palma, M., Venneri, M.A., Roca, C., and Naldini, L. 2003. Targeting exogenous genes to tumor angiogenesis by transplantation of genetically modified hematopoietic stem cells. Nat. Med. 9:789-795.

32. Santambrogio, L., et al. 2001. Developmental plasticity of CNS microglia. Proc. Natl. Acad. Sci.U.S. A 98:6295-6300.

33. Ohmi, K., et al. 2003. Activated microglia in cortex of mouse models of mucopolysaccharidoses I and IIIB. Proc. Natl. Acad. Sci. U. S. A. 100:1902-1907.

34. Crawley, J.N. 2000. What's wrong with my mouse? John Wiley and Sons Ltd. New York, New York, USA. 47-63.

35. Westerhof, G.R., et al. 2000. Comparison of different busulfan analogues for depletion of hematopoietic stem cells and promotion of donor-type chimerism in murine bone marrow transplant recipients. Cancer Res. 60:5470-5478.

36. Rezaie, P., and Male, D. 2002. Mesoglia \& microglia: a historical review of the concept of mononuclear phagocytes within the central nervous system. J. Hist. Neurosci. 11:325-374.

37. Hickey, W.F., and Kimura, H. 1988. Perivascular microglial cells of the CNS are bone marrow-derived and present antigen in vivo. Science. 239:290-292.

38. Tonra, J.R., et al. 2001. Comparison of the timing of acute blood-brain barrier breakdown to rabbit immunoglobulin $\mathrm{G}$ in the cerebellum and spinal cord of mice with experimental autoimmune encephalomyelitis. J. Comp. Neurol. 29:131-144.

39. Bruck, W. 1997. The role of macrophages in Wallerian degeneration. Brain Pathol. 7:741-752.

40. Mueller, M., et al. 2001. Rapid response to identified resident endoneurial macrophages to nerve injury. Am. J. Pathol. 159:2187-2197.

41. Kuhlmann, T., et al. 2002. Differential regulation of myelin phagocytosis by macrophages/microglia, involvement of target myelin, Fc receptors and activation by intravenous immunoglobulins. J. Neurosci. Res. 67:185-190.

42. Kreutzberg, G.W. 1996. Microglia: a sensor for pathological events in the CNS. Trends Neurosci. 19:312-318. 43. Mueller, M., et al. 2003. Macrophage response to peripheral nerve injury: the quantitative contribution of resident and hematogenous macrophages. Lab. Invest. 83:175-185.

44. Wada, R., Tifft, C.J., and Proia, R.L. 2000. Microglial activation precedes acute neurodegeneration in Sandhoff disease and is suppressed by bone marrow transplantation. Proc. Natl. Acad. Sci. U. S. A. 97:10954-10959.

45. Sangalli, A., et al. 1998. Transduced fibroblasts and metachromatic leukodystrophy lymphocytes transfer arylsulphatase A to myelinating glia and deficient cells in vitro. Hum. Gene Ther. 9:2111-2119. 46. Muschol, N., et al. 2002. Secretion of phosphomannosyl-deficient arylsulphatase A and cathepsin D from isolated human macrophages. Biochem. J. 368:845-853.

47. Matzner, U., Harzer, K., Learish, R.D., Barranger, J.A., and Gieselmann, V. 2000. Long-term expression and transfer of arylsulfatase A into brain of arylsulfatase A-deficient mice transplanted with bone marrow expressing the arylsulfatase A cDNA from a retroviral vector. Gene Ther. 7:1250-1257. 48. Matzner, U., et al. 2001. Bone marrow stem cell gene therapy of arylsulfatase A-deficient mice, using an arylsulfatase A mutant that is hypersecreted from retrovirally transduced donor-type cells. Hum. Gene Ther. 12:1021-1033. 